\title{
hClock gene expression in human colorectal carcinoma
}

\author{
LIYING WANG $^{1 *}$, BOZAN CHEN $^{2 *}$, YAPING WANG ${ }^{2}$, NING SUN $^{3}$, \\ CHAO LU $^{3}$, RUIZHE QIAN ${ }^{3}$ and LUCHUN HUA ${ }^{2}$ \\ ${ }^{1}$ Clinical Skills Learning Center, School of Basic Medical Sciences, Fudan University, Shanghai 200032; \\ ${ }^{2}$ Department of Surgery, Huashan Hospital, Fudan University, Shanghai 200040; \\ ${ }^{3}$ Department of Physiology and Pathophysiology, School of Basic Medical Sciences, \\ Fudan University, Shanghai 200032, P.R. China
}

Received January 9, 2013; Accepted July 9, 2013

DOI: $10.3892 / \mathrm{mmr} .2013 .1643$

\begin{abstract}
In this study, we aimed to investigate changes in the expression of human Clock (hClock), a gene at the core of the circadian gene family, in colorectal carcinomas (CRCs) and to discuss the possible effects. Previous studies have revealed that the disruption of circadian rhythms is one of the endogenous factors that contribute to the initiation and development of CRCs. However, the underlying molecular changes to the circadian genes associated with CRCs have not been explored. Immunofluorescence and quantitative polymerase chain reaction (qPCR) analysis of the hCLOCK protein and gene expression were performed in 30 cases of CRC. The hCLOCK protein was expressed in all specimens obtained from 30 CRC patients. Higher levels of hCLOCK expression were observed in human CRC tissues compared with the paired non-cancerous tissues. hCLOCK expression was significantly higher in poorly differentiated, or late-stage, Dukes' grade tumors and in $64.3 \%$ of tumor cases with lymph node metastasis. The hClock gene was expressed in all specimens. A significantly higher expression of $h$ Clock was found in human $\mathrm{CRC}$ cases compared with paired non-cancerous tissues. There was a strong positive linear correlation between hClock gene expression and protein expression in human CRCs. A strong positive linear correlation was also found between $h$ Clock gene expression and $A R N T, H I F-1 \alpha$ and VEGF expression in human CRCs. There was no significant correlation between hClock and Bak, Bax, Bid, tumor necrosis factor receptor I (TNFR I) and TNFR II. The circadian gene hClock was stably expressed in human colorectal mucosa and was important
\end{abstract}

Correspondence to: Dr Luchun Hua, Department of Surgery, Huashan Hospital, Fudan University, 12 Wulumuqi Middle Road, Shanghai 200040, P.R. China

E-mail:drhua@126.com

*Contributed equally

Key words: circadian rhythm, clock gene, hClock, colorectal carcinoma in regulating the expression of downstream clock-controlled genes. hCLOCK may interact with HIF-1 $\alpha /$ ARNT and activate VEGF to stimulate tumor angiogenesis and metastasis.

\section{Introduction}

The circadian gene network sets and regulates the circadian rhythms in organisms as oscillations with an approximate $24 \mathrm{~h}$ cycle (1). At the molecular level, circadian rhythms are controlled by the interaction between positive and negative feedback loops consisting of several key clock regulators $(2,3)$. A model encompassing a feedback system involving heterodimer transcriptional factors (Clock and Bmal1), two cryptochromes (Cry1 and Cry2), and three Period (Per1, Per2, and Per3) regulators has been widely described. Among these clock genes, $h$ Clock is at the core of the circadian gene family.

Previous studies have revealed that the disruption of circadian rhythms is one of the endogenous factors that contributes to the initiation and development of colorectal carcinoma (CRC). Krugluger et al reported that the expression of hPer1 mRNA in CRCs was lower than that found in normal cells (4). Wang et al detected that the expression of the hPer2 protein in CRC cells was lower than that in non-cancerous cells (5). Correlations between the decreased hPer2 level and the patient's age, histological grade and TNM stage were also detected. The human Clock (hClock) gene functions upstream of hPer2 to regulate its expression (5). Therefore, the circadian $h$ Clock gene may play a vital role in the development of CRC.

Hoffman et al observed that the hCLOCK protein expression level was approximately two times higher in breast tumor cells compared with that observed in the adjacent normal tissues and three times higher than that in normal breast cells (6). Alhopuro et al reported that $\sim 53 \%$ of hClock genes in CRC cells had point mutations in the DNA sequence (7). Oshima et al demonstrated that the expression level of hClock mRNA in CRC cells was significantly higher compared with that found in normal tissues (8).

$\mathrm{CRC}$ is one of the most commonly observed malignancies and is the leading cause of cancer-associated mortality worldwide (9). In 2011, 141,210 new CRC cases and 49,380 mortalities were predicted in the USA (10). In China, $\mathrm{CRC}$ is the fourth leading cause of cancer mortality in large 
cities and the fifth in more rural areas. However, in Shanghai, the incidence of CRC and its mortality rate ranked second and third, respectively, for females (11). As surgical approaches and conventional therapeutics have been incapable of fully controlling the outcomes of CRC, there is an urgent need to develop more effective treatments.

The circadian rhythm is interconnected with many aspects of cellular function, such as cell proliferation, migration and differentiation, and it also helps regulate the digestive system $(12,13)$. The molecular mechanism governing the development of CRC is a multiple factor and multistep process, yet the molecular changes underlying it remain unexplored. The core circadian gene, $h$ Clock, may participate in the development of CRC, but the correlation between them has not been reported.

In this study, immunofluorescent histochemistry and quantitative polymerase chain reaction (qPCR) were used to characterize the role of hClock in the development of human CRC. The correlation between the hClock gene and tumor-related genes Bak, Bax, Bid, HIF-1 $\alpha$, ARNT, tumor necrosis factor receptor I (TNFR I), TNFR II and VEGF were also examined. Based on our data, the expression of $h$ Clock in human CRCs and its possible effects were discussed. Our investigation may provide a new angle for understanding tumorigenesis and a new target for CRC treatment development.

\section{Materials and methods}

Tissue samples. In total, 30 specimens were obtained from the Tumor Tissue Bank of the Center of Pathological Research in the Institutes of Biomedical Sciences at Fudan University (Shanghai, China). The specimens were obtained from patients who had undergone a radical CRC resection between January 2007 and September 2008 from the General Surgery Department of Huashan Hospital Affiliated to Fudan University (Shanghai, China). The specimens were all obtained between 10:00 am-14:00 pm. The cancerous tissue was dissected from the cancerous mucosa while the paired non-cancerous tissue was obtained from the normal section of the colorectal mucosa of the same patient. All tumor tissues were pathologically confirmed as CRC and the patients did not receive any presurgical treatment nor exhibited any metastasis. Following surgical removal, samples were immediately preserved in liquid nitrogen and stored at $-80^{\circ} \mathrm{C}$. Complete medical records including the patient's age, tumor site, tumor type, histological grade and Dukes' stage were also obtained. This study was approved by the Medical Ethics Committee of Huashan Hospital, Fudan University, Shanghai, China. Written informed consent was obtained from the patients.

Immunofluorescent histochemistry. Frozen tissues were cut into $6 \mu \mathrm{m}$ sections and stored at $-80^{\circ} \mathrm{C}$. Sections were fixed at $4^{\circ} \mathrm{C}$ with $70 \%$ ethanol solution and blocked by $10 \%$ normal goat serum solution at $37^{\circ} \mathrm{C}$ for $60 \mathrm{~min}$. Subsequently, sections were incubated with goat anti-human hClock antibody (1:50, Santa Cruz Biotechnology Inc., Santa Cruz, CA, USA) as the primary antibody at $4^{\circ} \mathrm{C}$ overnight. Following a brief wash with PBS, secondary donkey anti-goat IgG fluorescent antibody (1:200; Invitrogen Life Technologies, Carlsbad, CA, USA) was added for $2 \mathrm{~h}$ at room temperature. Following several washes with PBS, the slides were mounted. Sections which were only incubated with $10 \%$ normal goat serum solution, served as negative controls. The image analysis software Image-Pro Plus (Media Cybernetics, Rockville, MD, USA) was used to measure the Integrated Optical Density (IOD), which is the cumulative OD of the positive immunofluorescent expression of a selected area and represents the total protein expression of that area.

RNA extraction and first-strand cDNA synthesis. Preparation of total RNA from tissue samples was performed using the TRIzol reagent (Invitrogen Life Technologies). The quantity of total RNA was determined using UV spectrophotometry and RNA integrity was assessed using agarose gel electrophoresis. First-strand cDNA was prepared with oligo-dT primers using a commercial cDNA synthesis kit (ReverTra Ace qPCR RT kit, Toyobo, Osaka, Japan). The cDNA was subsequently amplified for 33 cycles with specific primers for Bak, Bax, Bid, HIF-1 $\alpha$, ARNT, TNFR I, TNFR II and VEGF. Glyceraldehyde 3-phosphate dehydrogenase (GAPDH) served as an internal reference (Table I). Sequence data were analyzed using the basic local alignment search tool (BLAST) on the National Centre for Biotechnology Information (NCBI) website (http://www.ncbi.nlm.nih.gov).

PCR reactions containing SYBR-green were amplified on a Corbett Real Time PCR machine (Bio-Rad, Hercules, CA, USA). Following reverse transcription, the cDNA was diluted to $10 \mathrm{ng} / \mu \mathrm{l}$. The PCR reaction mixture contained $4 \mu \mathrm{l}$ of diluted cDNA, $1 \mu \mathrm{l}$ of $10 \mu \mathrm{M}$ primers $(0.5 \mu \mathrm{l}$ of upstream primers, $0.5 \mu \mathrm{l}$ of downstream primers), $10 \mu \mathrm{l}$ SYBR Green Realtime PCR Master mix (Bio-Rad) and $5 \mu \mathrm{l}$ of $\mathrm{H}_{2} \mathrm{O}$, into a final volume of $20 \mu \mathrm{l}$. The samples were subjected to 40 cycles of amplification (denaturation at $95^{\circ} \mathrm{C}$ for $15 \mathrm{sec}$, primer annealing for $30 \mathrm{sec}$ and extension at $72^{\circ} \mathrm{C}$ for $30 \mathrm{sec}$ ).

Following each qPCR, melting profiles were performed in order to rule out the possibilities of nonspecific PCR products and primer dimers. Data from the qPCR was represented by fluorescent intensity, $\Delta \mathrm{Rn}$, and number of cycles. $\Delta R n=R^{+}-R^{-}, R^{+}$refers to the fluorescent intensity of products at any time, $\mathrm{Rn}^{-}$refers to the systematic basic fluorescent intensity (the fluorescent intensity at the 6-15th cycles). The threshold was set to the intermediate value of $\log _{\Delta R n}$ vs. cycle function. The CT value stands for the number of cycles at the cross point of $\Delta \mathrm{Rn}$ vs. threshold. The higher the CT value, the greater the number of steady cycles, thus the lower the gene expression concentration. As the amplification efficiencies of the target genes and the internal control were equal, the relative changes to the target gene expression in the tumor cells compared with that in normal colorectal mucosa $(\Delta \mathrm{CT}$ calibrator value) were calculated using the equation $2^{-\Delta \Delta C T}$, where $\Delta \Delta \mathrm{CT}=\Delta \mathrm{CT}_{\text {(non-cancer) }}-\Delta \mathrm{CT}_{\text {(cancer) }}$. The $\Delta \mathrm{CT}$ values were determined by subtracting the average GAPDH CT value from the average target gene $\mathrm{CT}$ value.

Statistical analysis. Data are reported as the means \pm SD. Comparison between the groups was performed using the paired Student's t-test. Relative analysis was conducted using Pearson's $\chi^{2}$ test. IBM SPSS Statistics 19.0 was used for all data analysis. $\mathrm{P}<0.01$ was considered to indicate a statistically significant difference. 
Table I. Primer pairs used for qPCR.

\begin{tabular}{|c|c|c|}
\hline Gene & Primer $\left(5^{\prime}-3^{\prime}\right)$ & Product length (bp) \\
\hline hClock & $\begin{array}{l}\text { Forward: GGCTGAAAGACGACGAGAAC } \\
\text { Reverse: GGTGTTGAGGAAGGGTCTGA }\end{array}$ & 210 \\
\hline$A R N T$ & $\begin{array}{l}\text { Forward: CAAGCCCCTTGAGAAGTCAG } \\
\text { Reverse: GGGGTAGGAGGGAATGTGTT }\end{array}$ & 165 \\
\hline$B a k$ & $\begin{array}{l}\text { Forward: GGGTCTATGTTCCCCAGGAT } \\
\text { Reverse: AATGGGAGCAGGGGTAGAGT }\end{array}$ & 165 \\
\hline Bax & $\begin{array}{l}\text { Forward: TCTGACGGCAACTTCAACTG } \\
\text { Reverse: TTGAGGAGTCTCACCCAACC }\end{array}$ & 188 \\
\hline Bid & $\begin{array}{l}\text { Forward: GAGGATTGTGGCCTTCTTTG } \\
\text { Reverse: CTCAGCCCAGACTCACATCA }\end{array}$ & 185 \\
\hline$H I F-1 \alpha$ & $\begin{array}{l}\text { Forward: GAAAGCGCAAGTCCTCAAAG } \\
\text { Reverse: TGGGTAGGAGATGGAGATGC }\end{array}$ & 167 \\
\hline TNFR I & $\begin{array}{l}\text { Forward: GTGCCTACCCCAGATTGAGA } \\
\text { Reverse: TGTCGATTTCCCACAAACAA }\end{array}$ & 175 \\
\hline TNFR II & $\begin{array}{l}\text { Forward: GAGTTGTGGAAAGCCTCTGC } \\
\text { Reverse: CAGGGGGAGAAACAAACAAA }\end{array}$ & 193 \\
\hline$V E G F$ & $\begin{array}{l}\text { Forward: AAGGAGGAGGGCAGAATCAT } \\
\text { Reverse: ATCTGCATGGTGATGTTGGA }\end{array}$ & 226 \\
\hline GAPDH & $\begin{array}{l}\text { Forward: ACAGCCGCATCTTCTTGTGCAGTA } \\
\text { Reverse: GGCCTTGACTGTGCCGTGAATTTT }\end{array}$ & 226 \\
\hline
\end{tabular}

qPCR, quantitative polymerase chain reaction; TNFR, tumor necrosis factor receptor.

Table II. Correlation between increased hCLOCK protein expression levels in CRC and clinical-pathological features.

\begin{tabular}{|c|c|c|c|}
\hline $\begin{array}{l}\text { Clinical-pathological } \\
\text { features }\end{array}$ & $\begin{array}{l}\text { Increased hCLOCK } \\
\text { expression in tumor }\end{array}$ & $\begin{array}{c}\text { Non-increased hCLOCK } \\
\text { expression in tumor }\end{array}$ & P-value \\
\hline Gender & & & 0.039 \\
\hline Male & 8 & 11 & \\
\hline Female & 3 & 8 & \\
\hline Tumor site & & & $>0.05$ \\
\hline Colon & 7 & 11 & \\
\hline Rectum & 4 & 8 & \\
\hline Pathology type & & & $>0.05$ \\
\hline Ulcer & 6 & 11 & \\
\hline Protrude & 5 & 6 & \\
\hline Infiltrating & 0 & 2 & \\
\hline Histological grade & & & 0.042 \\
\hline $\mathrm{I}$ & & 3 & \\
\hline I-II, II & 7 & 13 & \\
\hline II-III, III & 4 & 3 & \\
\hline Dukes' stage & & & 0.032 \\
\hline 0 & 0 & 1 & \\
\hline A & 0 & 4 & \\
\hline $\mathrm{B}$ & 4 & 7 & \\
\hline $\mathrm{C}$ & 7 & 7 & \\
\hline
\end{tabular}


Table III. Correlation between the hClock gene and other tumor-related genes.

\begin{tabular}{lcccccrrr}
\hline Statistical significance & Bak & Bax & Bid & HIF-1 $\alpha$ & ARNT & TNFR I & TNFR II & VEGF \\
\hline $\mathrm{r}$ & 0.114 & 0.005 & -0.272 & 0.543 & 0.608 & 0.370 & -0.280 & 0.714 \\
P-value & 0.548 & 0.980 & 0.415 & 0.046 & 0.027 & 0.068 & 0.083 & 0.008 \\
\hline
\end{tabular}

\section{Results}

Immunofluorescent histochemical analysis of hCLOCK protein expression. To analyze expression of the hClock gene in CRC, we first examined the expression of hCLOCK protein in 30 paired colorectal cancerous and non-cancerous tissues using immunofluorescent histochemistry. For these 30 cases, hCLOCK protein expression in colorectal tissues exhibited three typical patterns. Type I (11 cases): the immunofluorescence was markedly stronger in the cancerous cells compared with that in the paired non-cancerous cells, particularly in the nucleolus and cytoplasm. Type II (17 cases): there were no significant differences in the immunofluorescence between the cancerous and non-cancerous cells. Type III ( 2 cases): the immunofluorescence was stronger in the non-cancerous cells compared with that in the paired cancerous cells (Fig. 1).

In these 30 specimens, the average cumulative OD of the hCLOCK protein in cancerous tissues was $5389.59 \pm 2385.20$ and that in paired non-cancerous tissues was $4558.34 \pm 1385.87$, $\mathrm{P}<0.05(\mathrm{P}=0.034)$. These results revealed that the hCLOCK protein was expressed in all specimens and a higher expression level of hCLOCK protein was found in human CRCs compared with that in the paired non-cancerous tissues.

Correlation between hCLOCK protein expression and clinical-pathological features in CRC patients. In order to analyze the clinical and pathological relevance of high hCLOCK protein expression in human CRC, we assessed the correlation between hCLOCK expression and the respective clinical-pathological parameters in the 30 cases of CRC. As shown in Table II, an increased expression of hCLOCK protein was found more in male than female patients $(\mathrm{P}=0.039)$. We observed a higher expression of hCLOCK in poorly differentiated $(\mathrm{P}=0.042)$, or late-stage, Dukes' grade $(\mathrm{P}=0.032)$ tumors. Furthermore, $64.3 \%$ of tumors with lymph node metastasis expressed higher hCLOCK levels. However, no correlations were found between high levels of hCLOCK expression and age, tumor site or pathology type.

Determination of hCLOCK $m R N A$ level by $q P C R$. We further analyzed hClock mRNA levels in the 30 paired cancerous and non-cancerous tissues by qPCR. The quantity of hClock mRNA was normalized to the endogenous reference gene, GAPDH. The normalized hClock mRNA expression $(\Delta C t)$ of tumorous tissues was subsequently compared with the $\Delta \mathrm{Ct}$ of the paired non-tumorous tissues from the same CRC patient in order to determine their relative expression levels $(\Delta \Delta \mathrm{Ct})$ (Fig. 2). The $\Delta \mathrm{Ct}$ of hClock in tumorous tissues $(5.91 \pm 1.36)$ was significantly lower than that found in paired non-tumorous tissues $(6.26 \pm 1.47$; $\mathrm{P}=0.002)$, indicating a significantly higher level of $h$ Clock expression in human CRCs than in paired non-cancerous tissues.
A

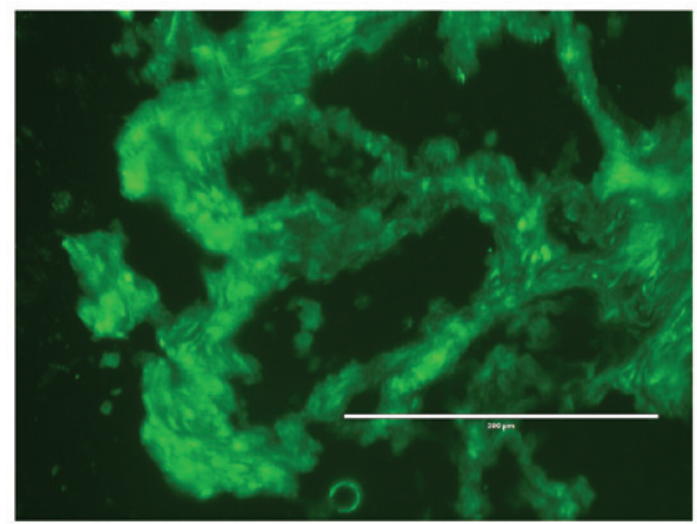

B

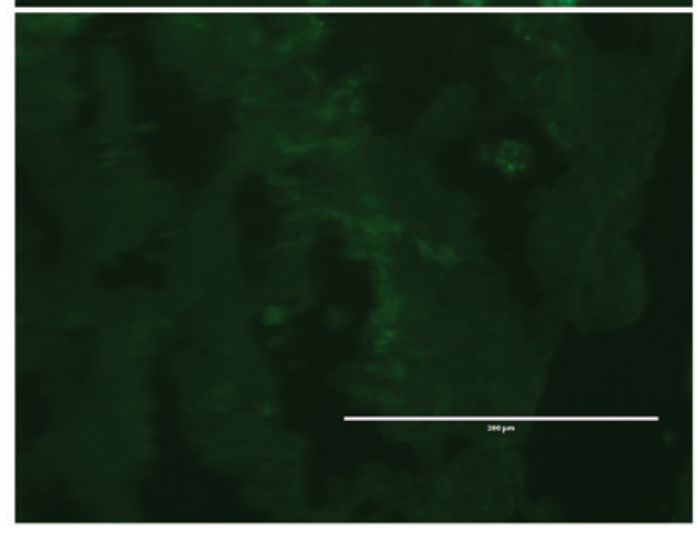

Figure 1. Immunofluorescent histochemistry of hCLOCK protein in human colorectal cancers. (A) Cancer tissue, (magnification, x40). (B) Paired normal mucosa tissue (magnification, $\mathrm{x} 40$ ).

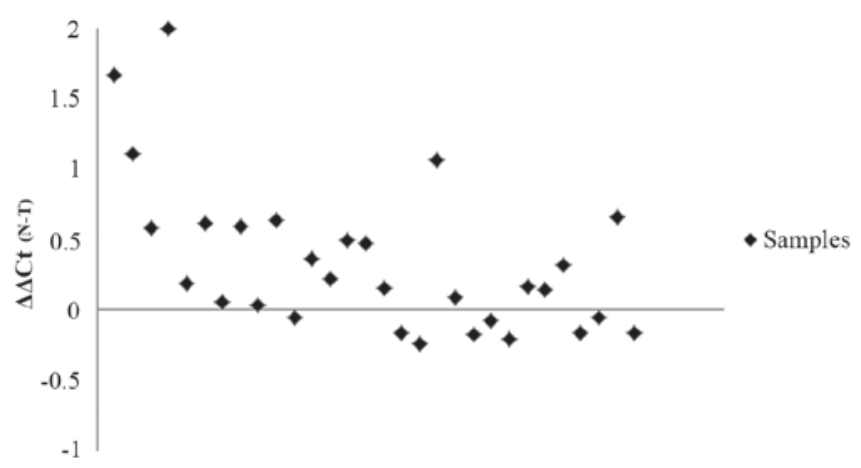

Figure 2. Distribution of quantitative polymerase chain reaction $\Delta \Delta \mathrm{Ct}$ of hClock mRNA in tumor and paired non-tumor tissues.

Amongst the 30 specimens, 12 tumor tissues demonstrated a high expression of the hClock gene. Furthermore, in these 12 tumor tissues, 10 specimens also demonstrated a higher expression of the hCLOCK protein. The correlation coefficient (r) between hClock mRNA and the hCLOCK protein was 
0.474, $\mathrm{P}<0.01(\mathrm{P}=0.008)$. The expression of the hClock gene and the hCLOCK protein revealed a strong positive linear correlation in human CRCs.

Correlation between the hClock gene and tumor-related genes. To further investigate the potential role of hClock at the molecular level, the correlation between the hClock gene and a number of tumor-related genes were determined by qPCR. As shown in Table III, there was a strong positive linear correlation between the $h$ Clock gene and the ARNT, HIF-la and VEGF genes in human CRCs. There was no significant correlation between hClock with Bak, Bax, Bid, TNFR I and TNFR II. The higher expression of ARNT, HIF- $1 \alpha$ and VEGF in poorly differentiated, or late-stage, Dukes' grade tumors was observed. Furthermore, high VEGF expression was found in 14 tumor cases with lymph node metastasis.

\section{Discussion}

It has been demonstrated that the circadian clock has important roles in orchestrating temporal integrations of physiology and behavior with the environment. This master clock, which resides in the hypothalamic suprachiasmatic nucleus in mammals, synchronizes multiple peripheral oscillators to ensure temporally coordinated physiology $(1,14,15)$. Clocks located outside the brain, peripheral clocks, are important in regulating physiological functions and pathological situations.

Previous studies have suggested that the disruption of circadian rhythms, which are regulated by circadian genes, was one of the endogenous factors that contribute to tumor initiation and progression. Studies have reported evidence regarding the beneficial effects of chronotherapy, which refers to the delivery of chemotherapy according to the circadian rhythms $(16,17)$. The hClock gene is at the core of the circadian gene family and directly, or indirectly, regulates certain clock-controlled genes $(18,19)$. The role of $h$ Clock in human CRCs is unclear. In this study, we aimed to investigate this question directly. We first examined the protein expression of hCLOCK in CRC patients using immunofluorescent histochemistry. The results revealed that hCLOCK was expressed in all CRC specimens and there was a higher expression of hCLOCK in human CRC tissues compared with that found in the paired non-cancerous tissues $(\mathrm{P}=0.034)$.

We further analyzed hCLOCK expression patterns with the respective clinical-pathological features of these colorectal tumor tissues. We found that cancerous tissues with poorly differentiated (grade II or above), or late-stage, Dukes' grade tumors (stage B or C) demonstrated increased expression of hCLOCK protein. The greater expression of hCLOCK in the CRC tissues of male patients compared with that in paired non-cancerous tissues was observed, but not in female patients. This may be further validated by using a larger sample set in the future.

qPCR analyses revealed that $h$ Clock was expressed in all of the specimens. A significantly higher level of hClock expression was found in human $\mathrm{CRC}$ compared with that in the paired non-cancerous tissue $(\mathrm{P}=0.002)$. hClock had a positive linear correlation with the hCLOCK protein in human CRCs $(r=0.474, \mathrm{P}=0.008)$. A higher expression of $h$ Clock was prevalent in tumor malignancies of a higher degree. These results suggested that the hClock is closely related to the development and prognosis of human CRCs.

We hypothesized that the circadian gene hClock may be a proto-oncogene. A proto-oncogene is usually a normal gene with rhythm, which is overexpressed and subsequently loses its rhythmic character in tumor cells. Hoffman et al observed deregulation of tumor-related genes in the hClock-silent cell strain, and that the protein hCLOCK expression in breast tumor cells was significantly higher compared with that in the adjacent normal tissues (6). Fu et al reported that c-myc in the tumorous cells of mice was regulated by circadian genes (20). The hClock gene correlated with cell cycles and had the characteristics of rhythmicity and specificity (21). This hypothesis requires further validation by examination with a larger sample set.

Circadian genes are important in regulating certain downstream clock-controlled genes (CCGs), including a variety of tumor-related genes (22). In our study, there was a significant positive linear correlation between the hClock gene and the ARNT, HIF- $1 \alpha$ and VEGF genes in human CRCs. There was no significant correlation between hClock and Bak, Bax, Bid, TNFR I or TNFR II. Malignant tumors promote the secretion of various proangiogenic factors, of which the most important is VEGF. VEGF has a marked proangiogenic function and is closely related to tumor differentiation, Dukes' stage tumors and tumors with lymph node metastasis.

When tumors were under environmental hypoxic conditions, the heterodimer HIF-l $\alpha /$ ARNT would be overexpressed, resulting in a higher VEGF expression. hClock may interact with HIF-1 $\alpha /$ ARNT and activate VEGF to stimulate tumor angiogenesis and metastasis $(23,24)$.

In conclusion, our data suggest that aberrant expression of hClock may correlate with the development and progression of CRCs. However, the mechanisms leading to a high expression of hClock in CRCs and how this promotes tumorigenesis remains unclear. Further studies using a larger sample set are required in order to elucidate the role of hClock in human CRCs.

\section{Acknowledgements}

This study was supported by the Natural Science Foundation of China (81070234 and 81000355).

\section{References}

1. Panda S, Hogenesch JB and Kay SA: Circadian rhythms from flies to human. Nature 417: 329-335, 2002.

2. Chen-Goodspeed M and Lee CC: Tumor suppression and circadian function. J Biol Rhythms 22: 291-298, 2007.

3. $\mathrm{Ko} \mathrm{CH}$ and Takahashi JS: Molecular components of the mammalian circadian clock. Hum Mol Genet 15: R271-277, 2006.

4. Krugluger W, Brandstaetter A, Kállay E, et al: Regulation of genes of the circadian clock in human colon cancer: reduced period-1 and dihydropyrimidine dehydrogenase transcription correlates in high-grade tumors. Cancer Res 67: 7917-7922, 2007.

5. Wang Y, Hua L, Lu C and Chen Z: Expression of circadian clock gene human Period2 (hPer2) in human colorectal carcinoma. World J Surg Oncol 9: 166, 2011.

6. Hoffman AE, Yi CH, Zheng T, et al: CLOCK in breast tumorigenesis: genetic, epigenetic, and transcriptional profiling analyses. Cancer Res 70: 1459-1468, 2010. 
7. Alhopuro P, Björklund M, Sammalkorpi H, et al: Mutations in the circadian gene CLOCK in colorectal cancer. Mol Cancer Res 8: 952-960, 2010.

8. Oshima T, Takenoshita S, Akaike M, et al: Expression of circadian genes correlates with liver metastasis and outcomes in colorectal cancer. Oncology reports 25: 1439-1446, 2011.

9. Zheng S and Cai SR: Colorectal cancer epidemiology and prevention study in China. Chinese-German J Clin Oncol 2: 72-75, 2003.

10. Siegel R, Ward E, Brawley O and Jemal A: Cancer statistics, 2011: the impact of eliminating socioeconomic and racial disparities on premature cancer deaths. CA Cancer J Clin 61: 212-236, 2011

11. Zheng S and Cai SR: Colorectal cancer epidemiology and prevention study in China. Chinese-German J Clin Oncol 2: 72-75, 2003.

12. Zhang EE, Liu AC, Hirota T, et al: A genome-wide RNAi screen for modifiers of the circadian clock in human cells. Cell 139: 199-210, 2009.

13. Scheving LA: Biological clocks and the digestive system. Gastroenterology 119: 536-549, 2000.

14. Reppert SM and Weaver DR: Coordination of circadian timing in mammals. Nature 418: 935-41, 2002.

15. Hirota $\mathrm{T}$ and Fukada Y: Resetting mechanism of central and peripheral circadian clocks in mammals. Zoolog Sci 21: 359-68, 2004.

16. Innominato PF, Focan C, Gorlia T, et al: Circadian rhythm in rest and activity: a biological correlate of quality of life and a predictor of survival in patients with metastatic colorectal cancer. Cancer Res 69: 4700-4707, 2009.
17. Block KI, Block PB, Fox SR, et al: Making circadian cancer therapy practical. Integr Cancer Ther 8: 371-386, 2009.

18. Gekakis N, Stakins D, Nguyen HB, et al: Role of the CLOCK protein in the mammalian circadian mechanism. Science 280: 1564-1569, 1998.

19. Matsuo T, Yamaguchi S, Mitsui S, Emi A, Shimoda F and Okamura H: Control mechanism of the circadian clock for timing of cell division in vivo. Science 302: 255-259, 2003.

20. Fu L, Pelicano H, Liu J, Huang P and Lee C: The circadian gene Period2 plays an important role in tumor suppression and DNA damage response in vivo. Cell 111: 41-50, 2002.

21. Polidarová L, Soták M, Sládek M, Pacha J and Sumová A: Temporal gradient in the clock gene and cell-cycle checkpoint kinase Weel expression along the gut. Chronobiol Int 26: 607-620, 2009

22. Gery S and Koeffler HP: The role of circadian regulation in cancer. Cold Spring Harb Symp Quant Biol 72: 459-464, 2007.

23. Park JE, Lee DH, Lee JA, Park SG, Kim NS, Park BC and Cho S: Annexin A3 is a potential angiogenic mediator. Biochem Biophys Res Commun 337: 1283-1287, 2005.

24. Chau NM, Rogers P, Aherne W, et al: Identification of novel small molecule inhibitors of hypoxia-inducible factor-1 that differentially block hypoxia-inducible factor-1 activity and hypoxia-inducible factor-1alpha induction in response to hypoxic stress and growth factors. Cancer Res 65: 4918-4928, 2005. 